\title{
A Preliminary Analysis of How a Pianist Expresses Emotions with Piano Performance
}

\author{
Jie BAI \\ No.18 of dianzi road Yanta District Xi'an Shaanxi province, Music Department of Xi'an \\ Shiyou University \\ 530299786@qq.com
}

\begin{abstract}
Key words:Piano performance, emotional expression, methods and techniques
\end{abstract}
\begin{abstract}
The piano performance is a kind of expressive language, and the combination of the emotional desire and the technical support can zchieve better performance.During the course of piano playing , pay attention to the combination of emotion and skills, will understand the works by understanding,based on the original presentation after the first composer active imagination on level second players active imagination,so that the modern individual spirit into the words full of new glory.In this article,the author analyzes and explores the necessary conditions of emotional expression in piano performance,and how to carry out emotional expression.

The piano is one of the most complex human art movement behavior, when the smooth operation of performing the work, must have fine control, on which the memory of body movements, skilled operation and adjust the action of mental process and the properties of these mental processes. Good psychological process on superb technology, ability is the necessary premise to master knowledge and skills, at the end of the day to see superb technical level depends on the basic qualities of the players, the height from players needed to achieve the basic literacy, solid basic skills is the primary condition, theoretical basis is the key to read music. If music is the language, then the background of the work is the context, and the language can be put into context to show its uniquebrilliance.
\end{abstract}

The composing background includes social environment, psychological state, composition time and creative motivation, and the theme of the event is the composer's specialty. In the German unification and outward expansion of 1801, Beethoven has set about writing the moonlight sonata (op. 27 no. 2) "and" rural sonata (op. 28, no. 15), compared with two the latter is more gentle soothing, less passion, interfered the former ups and downs of emotion and mood in the latter. In 1796-1800, Beethoven's ear deep influence on him, these days he hearing decline, internal organs also tortured, tortured in his body, his mind and composing style also become more mature, with Julie, keisuke check after the relationship ended in failure, under the music is grieving pain but also full of love and passion of "moonlight sonata". And rural sonata is one of writer's voices to Vienna, Beethoven's piano works, mountain stream, jungle, peak valley is the source of his inspiration, music line is fluent beautiful, happy and funny music tone, two themes interweave mutually beget each other.

We are not able to clear a composer's style is fixed, Hoffman once said: "there is a fallacy that is the name of the composer to inference, Beethoven must think so, and Chopin must play like that... "But music works are structured in a certain period, according to the typical elements of music theme, music language and formal structure we can is divided into" baroque "' classical period" "romantic period", etc.; Objectively speaking, music creation needs the author based on the understanding of the current life, out of need or emotional or reality, combined with the creative impulse, seize the fleeting inspiration, using music art technique of expression, around the theme display and image shaping a series of separate primary and secondary music material weight, reasonable and symmetrical arrangement and organization, make its should both comply with the regularity of life, labor, and meet the requirements form of works of music genre, become music works with the aesthetic structure, to achieve the perfect harmony of art. Score as a carrier of the music works, constitute a necessary piece of clef, key signatures, shoot number, rhythm, speed, and so on all detailed annotation on reproduction, and great article notes is the creator ways to convey 
information, notes and chords became the basic elements of propping up the whole piece of music, that the foundations of the basic elements like when building high-rise buildings, there is no solid is rigorous, the basis of the error is in the know note can't afford to build high-rise buildings, unable to create exciting tune.

Condenses into music composer's creative thinking, which in turn in the structure of the form of music works, melody, rhythm, melody is the elements of music performance, so before playing it, need to music works make a correct understanding and reasonable division of phrase, to prevent speculation, avoid misunderstanding. Just think, if there is no pause in music works, no breath, no clauses, became showed no mechanical notes beating, it's direct loss of the inner beauty of music emotion further music, and where to talk about? After fully understanding of the creation background, therefore, to try to understand the author's creation intention, music experience motivation, express the content, the theme of the music, like a sentence is a mental state of map symbols, by the basic elements of some notes, chords and habits according to certain rules combined into a melody as a whole, so the players must be expressed in understanding the content after the phrase can be a preliminary division, in a reasonable way to play. And at the same time, need to know has music and poetry, music thoughts in music to keep their own characteristics, meaning, so in practice the piano works, players can according to the form structure, harmony, voice analysis, melody line ups and downs to classify the phrase; Players, on the other hand, still need to analyze sing s style of play, traces the development of music: sonata at the beginning of the first movement is only one theme, gradually developed into two different tone and the theme of the associated with each other, since then determined the classical sonata mainly according to the "fast, slow, fast" three movement, this format to adapt to the era of gout, at that time of the first artists to make the audience interested in every part of a piece, rather than any part of the individual, can be said to be "music" for public, le purport motivation has formed a single fixed form; After the theme development of Haydn, the melody of Mozart was broad and full. Beethoven's first reform of the sonata was to overturn its rigid pattern, to give the freedom of the content of the relationship, so that the sonata would exert its eloquence. He's 32 sonatas in thirteen is the first four movement, 13 in the first three movements, six are two movement, tone and the theme of freedom of choice, Beethoven sonata typical into flexible tool for express emotions, in the traditional sonata mode combined with the final chapter, every movement can have different themes. Music works is the composer as a medium of emotional communication, is also the spirit of time, players through the methods of literature research, summarize the experience and observation analysis methods, such as s works and its relevant information such as region, historical background, such as whether the title music, observes, to extract the required information for his piano playing.

By understanding the intention of the works and the accuracy of the sentences, the players can improve the quality of the playing to a great extent, and also make the emotional expression of the expression have a solid foundation.

The piano as a delicate multi-vocal instrument, the music of the music involved in the complex of music knowledge is not to be repeated. Players either analysis expression form and understand music or read the direction of the term, etc., all and music theory knowledge is closely linked, so learning music theory knowledge not only won't let players feel isolate, instead, to boost the role playing, the piano works will have a wide variety of expressions terms, because of the inner feelings and ideas expressed by music will be involved in the process of music emotion, and this was ups and downs of emotion that can use the texture in the piano, harmony, pitch, intensity, speed and other forms of details, a performer in a preliminary reading, find out the author's creation intention and emotion, the expression that can be according to the spectrum on the marked term play to a more accurate expression of the music content and purpose. Various expression term have their range of content: the term Presto or Grave, as shown in the beginning of each piece of music, sets the tone and rhythm of the whole piece from its inception. A variety of musical notes, such as the conjoined and the stamped symbols, can be used to drive the atmosphere, light and heavy, or quiet and passionate; The various kinds of grace notes as the indispensable means of the musicians can reflect the little nifty of flexibility; Gradually stronger and weaker markers can be used to 
express the emotion gradually intense or the mood calm; Repeated markers can highlight the theme again, and music's world has too many terms, such as this one. When the performer has grasped these expressions, the initial emotions of the music are naturally mastered. Such as facial expressions if we only see on the surface of the spectral term, the spectrum on the surface of the notes are removed, we can find that different or sign with the feelings of the connection of the former after bearing into a rise the line of ups and downs, has become a piece of music of meridians, makes the work of music image more vivid.

Music has long been in a special position because of its various playing methods. Play is a kind of special complex movement skills, which requires our fingers or quick or slow play the notes sound, beauty of the music and music intentions, it will need a good other related muscle coordination and technique are gradually formed in the long-term practice of people, the formation of any technology has its own objective regularity.

Five fingers to practice is the foundation of the whole piano technology, flexible and powerful players training, sensitive and listen to the command of fingers need to do the basic exercise, such as use of basic practice teaching material, finger like Hanon play big minor scales arpeggios and essential to the slow practice, and so on. When do the basic exercise, no matter use staccato or legato playing, all will be great help for fingers, staccato play is the most basic weight method, can help solve the problem of can't relax your shoulders and back to play, set up the concept of play with weight experience plays a rock-solid weight at the same time, the expressive voice quality, in the finger exercises support wrist more flexible; Legato method is used to play the effective training of weight transfer between the fingers, arms, play every sound must pay attention to the arm relaxed, in a loose a send to exercise between support and fingers of the finger muscles ability, tone will be more flexible, both continuous feeling and sense of melody.

For art "want to understand from the inside", if we think narrowly defined concept of music as music of the nature of "aesthetics", we will find that this definition is too limited to ancient Greece, or even the reason in the background of the Renaissance. Piano playing is a special art, from appreciating ways, those who had no formal music education of the audience were no formal music education of the audience is poor, because of the appreciation of piano performance description does not need any professional term, her value and practice the art of law by the given by the players, players improve its own value to maximize the value of the music.

It is an important mission of the performer to create two degrees in any piano performance. As an important category in aesthetics, artistic conception of Chinese traditional culture summed up in "feeling" and "enlightenment" this kind of cognitive process, as a person, understanding music like players, two players for a long time in literature, art, philosophy, humanities, music and performance skills in the aspects of the accumulation, will be lifetime, players feeling music not only let themselves get sublimation, also make notes from the dead to have the change of life, this will be played through a series of change. Let music show with the what of the players, the new capacity, from the creator of the "narrative" into the "narrative" of the players, that cause the audience's emotional resonance, the second creation is performer diligence, wisdom, perceptual and rational synthesis reaction, however, this second writing is not an act of a pianist can finish, are the players in every play never stopped writing, practice, make this creative behavior habits can make success playing the piano.

Any pianist who plays piano works makes his music expressive, so that the audience is affected by the charm of music without emotional expression. Different genres, different times, different composer has its different characteristics, for players to grasp the elements in the works reproduce only when players to learn music comprehensive ability, knowledge background, understand the work, have the corresponding technical level, humanities knowledge accomplishment, for channels, to express show interpretation on the second creation thought emotional expression of the process. In to investigate the emotional expression of piano performance, the process, even with a professional mentor's guidance careful teachings, but because of its own knowledge storage is relatively poor, described in the thesis deep, still have a large part of the breadth and depth, this article from the basic qualities of a performer, music theory knowledge and emotional expression 
way of three aspects, in order to study the piano, topic and also sincerely hope that with the popularity of the piano, with the correct education idea, let more people enjoy the art, to go down on the keyboard music.

\section{Reference}

[1] Josef Hofmann. Analysis on piano playing [M]. People Music Press, 2000.

[2] Enrico Fubini. Western music aesthetic history [M]. Hunan Literature and Art Press, 2005.

[3] Wang Chaogang. New theory of instrumental performance teaching [M]. Shanghai Music Press, 2005.

[4] XieJiashan. Music's "context" -- a kind of music hermeneutic field [M]. Shanghai Conservatory of Music Press, 2005.

[5] SergeySkribokov.The artistic principle of music style [M].Central music college press, 2008.

[6] Jiang Tao. How to express emotion better in piano performance [J]. Popular Literature and Art, 2016.

[7] Charles Rosen. Piano notes - the world of pianists [m.]. Modern press, 2010.

[8] Stephen Davis. Significance and performance of music [M.]. HunanLiterature and Art Press, 2007.

[9] Clarence G. Hamilton. Piano touch and expression [M.]. People Music Press, 1995.

[10] Li Xiaosi. How to improve the performance of music in piano playing [D.].Press of Harbin Normal University, 2016.

[11]Fu lei, Fu cong. Fu lei and Fu congtalk about music [M.]. Unite press, 2006.

[12]Wu Tieying, Sun Mingzhu. Concise piano teaching method [M.].Huale Press, 1997. 